\title{
Traduire
}

Revue française de la traduction

\section{La place de la traduction et de l'interprétation au sein des services publics espagnols : historique, situation actuelle et perspectives}

\section{Carmen Valero Garcés}

Traducteur : Géraldine Galeote, Rosalía Barcia, Anne-Charlotte Chasset, Béatrice Cornière, Aurélie Dubois, Pauline Louvet et Viviane Petit

\section{(2) OpenEdition} Journals

Édition électronique

URL : http://journals.openedition.org/traduire/484

DOI : $10.4000 /$ traduire.484

ISSN : 2272-9992

Éditeur

Société française des traducteurs

Édition imprimée

Date de publication : 15 décembre 2012

Pagination : 77-92

ISSN : 0395-773X

Référence électronique

Carmen Valero Garcés, « La place de la traduction et de l'interprétation au sein des services publics espagnols : historique, situation actuelle et perspectives », Traduire [En ligne], 227 | 2012, mis en ligne le 01 décembre 2014, consulté le 19 avril 2019. URL : http://journals.openedition.org/traduire/484 ; DOI : 10.4000/traduire.484 


\section{La place de la traduction et de l'interprétation au sein des services publics espagnols : historique, situation actuelle et perspectives}

\section{Carmen Valero Garcés}

\section{Introduction}

En 1998, j’ai rédigé un article intitulé "¿Traducción e interpretación en los servicios públicos? ¿De qué me hablas? ¿Una nueva especialización? „(1) dans le cadre des Troisièmes rencontres de la traduction qui se déroulèrent à l'Université d'Alcalá de Henares (Madrid) avec pour thème : nouvelles tendances et applications de la traduction(2). Cette communication faisait figure d'OVNI au milieu de travaux qui portaient sur les avancées technologiques et sur leur application concrète ainsi que sur l'influence des études en matière de traduction. Elle faisait également état de recherches portant sur les difficultés liées à la traduction de textes spécialisés de l'anglais, du français ou de l'allemand vers l'espagnol, ainsi que des stratégies pour traduire des aspects culturels entre les langues de travail proposées par les cursus universitaires espagnols ou que réclamait le marché du travail. Pour ma part, je m'aventurais à aborder une réalité émergente en Espagne avec l'arrivée massive de personnes parlant des langues quasi inconnues (l'arabe, le roumain, le polonais, le bulgare, l'urdu, le bambara, le swahili) et de cultures différentes. Avec ces nouveaux besoins à couvrir, surgissaient de nouvelles problématiques auxquelles il fallait - et il faut encore - faire face. II s'agissait de besoins de tous types (éducatifs, économiques, sociaux, politiques, humanitaires) qui appelaient également des solutions diverses : prise en charge sociale, décisions politiques ou aide linguistique, pour n'en citer que quelques-unes.

Dans le domaine de la communication, le profil du traducteur et de l'interprète de conférence ainsi que celui du travailleur social étaient déjà bien consolidés; des centres de formation institutionnels dans ces domaines avaient déjà vu le jour (l'Espagne compte au moins 24 facultés de traduction et d'interprétation). En revanche, ni dans un cas ni dans l'autre, les besoins des nouveaux arrivants n'étaient couverts par les professionnels en poste, en partie

(1) Traduction et interprétation dans les services publics. De quoi me parles-tu ? Une nouvelle spécialité ?

(2) Valero Garcés 1998 ; 267-277. 
du fait du problème linguistique et culturel : comme ils ne parlaient pas la même langue et/ou ne partageaient pas la même culture, la communication devenait extrêmement difficile. Dans l'article de 1998 susmentionné (Valero Garcés 1998 : 267-277), je faisais référence à cette réalité et au fait qu'il fallait une nouvelle approche voire une redéfinition des concepts de " traduction " et " d'interprétation ".

Depuis lors, plus de dix ans se sont écoulés. Les réponses apportées ont été nombreuses et les questionnements se sont également multipliés, tant au niveau national qu'international, dans un contexte chaque fois plus ancré dans la mondialisation. Cette réalité était déjà connue depuis longtemps dans d'autres pays (Australie, Canada, USA, Royaume-Uni, Allemagne, France). En Espagne, les populations immigrantes sont d'abord arrivées au compte-gouttes, à partir de 1986 et de l'entrée du pays dans l'Union européenne, puis le phénomène a connu une véritable explosion, en Espagne comme dans d'autres pays de la Communauté.

Quelques brefs rappels des moments clés du développement de la Traduction et de l'Interprétation dans les Services Publics (TISP) au niveau international nous aideront à nous faire une idée plus précise de la situation espagnole.

Je mentionnerai tout d'abord la création de l'organisation Critical Link (http://www.criticallink.org) au Canada en 1992 avec pour objectif l'organisation d'une rencontre internationale qui a eu lieu en 1995. Ce fut le premier congrès international consacré à la TISP (ou community interpreting). L'objectif principal était de comparer et d'échanger des expériences, des formations et des initiatives avec les délégués du monde entier qui travaillaient dans ce domaine(3). Critical Link est ainsi devenu le plus grand et plus ancien forum international sur la TISP. Les thèmes abordés lors de ses différents congrès reflètent l'évolution qui a touché non seulement la communication avec les populations immigrées, dans la plupart des cas dans des langues minoritaires, mais, plus généralement, l'ensemble de la société.

Dans le même temps, d'autres associations et d'autres forums ont vu le jour. Des séminaires, des congrès, des journées d'étude de tous types ont été organisés au niveau mondial pour échanger des expériences, étudier les nouvelles sociétés multiculturelles ou le développement des relations entre les populations étrangères et autochtones.

C'est en 1998, grâce à l'Université d'Alcalá de Henares, que l'Espagne aborde pour la première fois la TISP. Depuis lors, nous avons continué à travailler dans cette perspective avec l'organisation, en 2002, des Cinquièmes rencontres de la traduction devenues le Premier congrès international sur les TISP entièrement consacré à ce champ d'étude et dans la lignée de Critical Link, autour du thème : de nouveaux besoins pour de nouvelles réalités(4). L'objectif était

(3) Ce congrès fut suivi par plusieurs autres, dans divers endroits et à différentes dates (à Vancouver en 1998, Montréal en 2001, Stockholm en 2004, Sydney en 2007, Birmingham en 2010 et le prochain se tiendra à Montréal en 2013).

(4) Valero Garcés et al. 2002. 
d'offrir à tous les acteurs ayant un rapport direct ou indirect avec la traduction et l'interprétation un lieu d'échange autour de la nouvelle réalité sociale qui se dessinait en Espagne. Le débat tourna autour de la complexité de la tâche de l'intermédiaire ou troisième chaînon appelé médiateur, interprète ou traducteur - en tant qu'instrument de liaison dans les services publics (dispensaires, hôpitaux, écoles, ONG, bureaux de l'État, etc.). II fut question de sa formation et de son degré de professionnalisation réel et souhaitable ainsi que de l'influence de facteurs exogènes à l'activité du transfert de l'information.

Trois ans plus tard, en 2005, a été organisé le Deuxième congrès international sur les TISP avec pour thème cette fois-ci la traduction comme médiation entre les langues et entre les cultures(5), afin de poursuivre le travail de dialogue et l'échange d'expériences et de projets engagés en 2002.

Nous voulions également nous faire l'écho du débat sur le rôle du traducteur et de l'interprète face à ces nouveaux défis. Notre idée de départ était que la traduction - prise dans son sens le plus large comme le transfert d'un message d'une langue à l'autre - continue de jouer un rôle fondamental dans la formation de nouvelles sociétés. Les différentes manières de comprendre ce transfert et le rôle joué par divers facteurs indépendants de la langue (les politiques nationales et internationales, les relations de pouvoir, les questions de genre, la reconnaissance des minorités culturelles, la position du traducteur et de l'interprète (T\&l) ou encore le(s) rôle(s) que doit jouer ce troisième chaînon de la communication) y étaient abordés. On a également analysé des propositions qui marquaient une avancée dans ce domaine et lancé la création du Réseau National Interuniversitaire COMUNICA(6), dont il sera question plus loin.

En avril 2008, a eu lieu le Troisième congrès international sur les TISP autour du thème Recherche et pratique dans la TISP : défis et alliances(7). Ce thème représentait un pas supplémentaire dans l'évolution et la reconnaissance de la profession de la T\&l dans les services publics, mais il s'agissait également d'un appel à la coopération et au travail d'équipe de toutes ces institutions (professionnels, formateurs ou acteurs de terrain en tout genre qui, d'une manière ou d'une autre, interviennent dans le processus de communication avec des personnes qui ne partagent pas leur langue et/ou leur culture et dont le but est de favoriser la consolidation d'une nouvelle société multiculturelle encore en construction).

En avril 2011 a été organisé le Quatrième congrès international sur les TISP où il a été question, cette fois, de "I 'avenir dans le présent : traduction et Interprétation dans les Services Publics dans un monde INTERcoNNEcTé(8) ", c'est-à-dire la TISP sur INTERNET. En écho aux change-

(5) Valero Garcés et al. 2005.

(6) Observatoire Permanent de la Communication entre les langues et les cultures en Espagne (red-comunica.blogspot.com).

(7) Valero Garcés et al. 2008.

(8) El futuro en el presente: Traducción e Interpretación en los Servicios Públicos en un mundo INTERcoNEcTado. 
ments dans nos sociétés actuelles, les travaux ont présenté l'application des Technologies de I'Information et de la Communication (TICS) à la TISP(9). Finalement, et d'une manière générale, les thèmes abordés lors des trois congrès consacrés jusqu'à présent à la TISP à l'Université d'Alcalá de Henares ont reflété l'évolution et la situation de la communication avec des populations étrangères, telles que je vais tenter maintenant de les expliquer. Pour cela, je vais évoquer l'évolution de la communication avec ces nouvelles populations dans trois domaines différents mais complémentaires, à savoir :

- le champ académique,

- le terrain professionnel,

- le domaine de la recherche,

et cela, selon trois points de vue différents qui correspondent aux trois niveaux d'intervention dans le processus de communication :

- les fournisseurs de services,

- les usagers,

- les intermédiaires ou personnes qui rendent possible la communication lorsqu'il s'agit de langues et de cultures distinctes.

En avril 2014 aura lieu le Cinquième congrès international sur les TISP.

Dans la suite de cet article, et afin d'en faciliter la compréhension, j'aborderai tour à tour les deux grands aspects suivants :

1. Évolution de la communication avec les populations étrangères dans le champ académique, le terrain professionnel et celui de la recherche.

2. Configuration de la communication : le rôle des fournisseurs de services, des usagers et les intermédiaires dans le processus de communication.

Je laisserai de côté le débat sur la dénomination et les limites encore imprécises(10).

(9) Par exemple, la mise en place et le développement de l'interprétation à distance en visioconférence, l'interprétation téléphonique ou l'application de la téléphonie mobile à la TISP, la formation des traducteurs et interprètes basée sur de nouvelles technologies, la recherche et l'expérimentation de solutions interdisciplinaires permettant d'améliorer la qualité et l'efficacité des traductions grâce aux nouvelles technologies, et les avancées dans la recherche conjointe de solutions entre institutions publiques et éducatives.

(10) Sur ce point, je renvoie à Pöchhacker (2002: 125-140) qui nous offre une description précise des limites de cette " activité imprécise " en soulignant qu'il s'agit d'une activité qui ne peut se limiter ni à des institutions spécifiques, ni à des langues ou des groupes culturels précis, du fait de la grande variété de situations institutionnelles et de bagages culturels auxquels l'on peut être confronté. Et tout comme Pöchhacker, j'estime que c'est cette extrême complexité du rôle de l'intermédiaire qui rend presque impossible sa définition. 


\section{L'évolution de la communication avec des populations étrangères dans trois domaines distincts}

Holly Mikkelson (2001) nous offre une radiographie précise du développement de la TISP. Selon cette auteure, les phases d'évolution auxquelles l'on assiste dans les situations semblables à celles connues en Espagne et dans d'autres pays de l'Union européenne, lors des dernières décennies, se présentent comme suit :

Phase 1. Désordre sur le marché et forte concurrence parmi ceux qui pratiquent cette activité, aggravée, dans le cas de la TISP, par la méconnaissance de la part des fournisseurs de services du rôle du T\&l et par la sous-estimation de son travail. Dans cette situation, les possibilités de formation et de promotion sont peu nombreuses, ce qui fut déjà le cas dans les années 1990, tandis que naît progressivement, chez les acteurs de terrain, le désir d'un travail meilleur. Les fournisseurs assistent alors au départ des meilleurs T\&l, ce qui déclenche alors la deuxième phase.

Phase 2. La consolidation de la profession et le développement d'un consensus sur les aspirations des acteurs de terrain. Pour y parvenir, l'on a recours à des programmes de formation et les T\&l commencent à se regrouper, alors que les institutions exigent dans le même temps des services de qualité.

Phase 3. Les associations regroupant les personnes qui sont sur le terrain commencent à édicter des règles de conduite, dont le respect obligatoire apporte progressivement prestige et reconnaissance au groupe.

Phase 4. Le respect de ces règles et le contrôle de l'accès à la profession signalent la professionnalisation de l'activité.

Le passage de la phase 1 à la phase 4 n'est pas sans heurts, et ne se réalise pas dans un laps de temps défini. II s'agit plutôt d'un processus circulaire qui se répète selon des modalités différentes en fonction des pays et des services concernés. J'en veux pour preuve la très grande diversité entre les pays dans le recrutement, la formation et l'accréditation des T\&l qui travaillent ou collaborent avec les services publics. Ainsi, certaines organisations offrent des services multilingues gratuits et d'autres les font payer. Certains gouvernements financent les services de TISP (Australie, Canada, Suède) alors que d'autres apportent un soutien indirect (RoyaumeUni, Belgique, Pays-Bas) ou les ignorent presque totalement (Italie, Espagne, Portugal et France). De la même manière, un cursus universitaire a été mis en place dans certains pays alors que dans d'autres il n'existe que des ateliers, des journées d'études ou des formations avec un nombre variable d'heures.

Cette situation très hétérogène rend difficile, selon Pöchhacker(11), le maintien de "l'unité dans la diversité ".

(11) $2002: 125-140$. 
Pour analyser l'évolution de la TISP dans les trois domaines indiqués (universitaire, professionnel et la recherche) à partir de l'expérience espagnole, le rapport du Réseau COMUNICA est sans doute le plus complet. Ce réseau a été créé en 2005. II réunit différents groupes de chercheurs et de formateurs en T\&l sur l'ensemble du territoire national et il a pour principal objectif d'être un Observatoire permanent de la communication entre les langues et entre les cultures, en abordant le domaine de la TISP de manière critique et engagée.

Le Réseau Comunica remplit trois grandes fonctions, qui sont :

1/ l'étude et l'analyse de l'évolution de la TISP sur l'ensemble du pays,

2/ la promotion de la professionnalisation de la TISP,

3/ la sensibilisation des responsables des services publics sur la nécessité d'une TISP de qualité.

Les membres du Réseau ont chacun une aire géographique d'étude, qui correspond la plupart du temps à la Communauté autonome dans laquelle se situe leur université, et ils couvrent, à eux tous, l'ensemble du territoire national. À partir de chacune de ces zones géographiques de référence, nous menons à bien une étude et une analyse de la réalité de la TISP, en essayant d'englober ces trois fonctions. Les résultats de cette analyse sont régulièrement divulgués lors de conférences, de congrès et de réunions nationales et internationales, ainsi que dans des articles et des publications monographiques des membres du Réseau.

Le travail du Réseau COMUNICA a également vocation à émettre une critique constructive et à promouvoir l'engagement social et, par le biais du blog du Réseau (http://red-comunica.blogspot.com), nous relayons les initiatives nationales et européennes entreprises dans ce domaine, les réunions d'experts qui ont lieu dans d'autres pays, ainsi que les publications sur ce sujet. Nous faisons également apparaître sur ce même blog les initiatives de formation mises en place dans le pays et dénonçons les situations de précarité dans la TISP qui portent atteinte aux droits fondamentaux (par exemple, en adressant des mémos à la presse nationale ou en intervenant comme témoins dans le cadre de plaintes déposées pour manquement au droit d'accès aux services d'un traducteur-interprète).

La formation est l'un des piliers essentiels de la professionnalisation de la TISP, au-delà de l'accréditation et d'une offre généralisée de ce service. En Espagne, où ce type de communication avec des populations étrangères est en voie de consolidation, l'on assiste depuis une dizaine d'années au lancement de plusieurs propositions de formation, celles-ci devant leur existence davantage à des organismes agissant de façon informelle qu'à l'Université en tant que lieu d'enseignement institutionnel.

Plus généralement, et comme cela se produit dans la plupart des pays de l'Union européenne, l'Espagne s'occupe actuellement de la mise en place définitive des nouveaux diplômes universitaires conformément aux exigences de l'Espace Européen de l'Éducation Supérieure (EEES). 
Jusqu'à présent, les licences de Traduction et Interprétation en Espagne n'offraient pas de formation spécifique en TISP, à de très rares exceptions près. Certaines incluaient spécifiquement quelques réflexions, remarques ou TD dans les cours d'interprétation consécutive et relationnelle, laissés à l'appréciation des chargés de cours. Le processus actuel de réforme de l'Enseignement Supérieur a offert à l'Université la possibilité d'inclure un contenu de formation en TISP dans les diplômes de deuxième cycle de Traduction et Interprétation. Toutefois, I'on observe que les voies empruntées ont été plutôt généralistes, limitées et marquées par des réglementations et des intérêts divers sans que cette nouvelle spécialité de T\&l, que l'on appelle TISP, soit prise en compte. II convient de renforcer la formation spécialisée de master, en troisième cycle, mais pour cela il faudra attendre.

En ce sens, I'Université d'Alcalá de Henares ferait figure de modèle avec son Master Universitaire Européen en Communication Interculturelle, Interprétation et Traduction dans les Services Publics(12), qui fait partie du réseau de Master Européen en Traduction (EMT Network)(13), reconnu par l'Union européenne, et présentant 9 combinaisons linguistiques, parmi lesquelles des langues minoritaires fortement représentées dans la zone centrale de l'Espagne où se trouve l'Université d'Alcalá de Henares. Ces combinaisons de langues sont d'une part l'espagnol et d'autre part l'allemand, l'arabe, le bulgare, le chinois, le français, l'anglais, le polonais, le roumain et le russe.

Les enseignements sont organisés à partir d'une perspective générale et pluridisciplinaire qui allie :

- l'application d'un modèle plurilingue et pluriculturel, avec d'éminents professionnels et des universitaires reconnus, de langues et cultures différentes, ainsi que des représentants d'institutions diverses, qui participent à des débats, conférences et ateliers à échelle réduite, en fonction des deux langues choisies ;

- la participation active des étudiants et l'échange d'expériences liées à la traduction et à l'interprétation dans différents domaines ;

- la pratique de l'interprétation et/ou de la traduction dans les deux langues choisies avec des textes réels tirés de situations vécues ;

- les stages dans des institutions publiques, pour permettre aux étudiants de découvrir le monde du travail ;

- la rédaction d'un mémoire de Master ou d'un projet de recherche qui forme l'étudiant à la recherche documentaire, à la préparation de projets et à la collaboration avec les collectifs d'institutions diverses.

(12) Máster Universitario Europeo en Comunicación Intercultural, Interpretación y Traducción en los Servicios Públicos http://www2.uah.es/traduccion

(13) http://ec.europa.eu/dgs/translation/programmes/emt 
L'objectif principal est de former des professionnels en communication interculturelle. Pour y répondre, on tente de fournir aux étudiants d'un côté les connaissances théoriques requises et de l'autre, les compétences, techniques et outils nécessaires pour agir en tant qu'intermédiaires linguistiques et culturels entre le personnel des institutions médicales, administratives et éducatives et des usagers ne maîtrisant pas la langue espagnole.

Le programme compte $60 \mathrm{ECTS}(14)$, répartis en trois modules :

- Communication interlinguistique

- Traduction et interprétation dans le domaine de la santé

- Traduction et interprétation dans le domaine légal et administratif.

Sur cet ensemble, plus de $50 \%$ (33 ECTS) sont propres à chaque combinaison linguistique, comme le montre le tableau ci-après qui présente les principaux cours et les ECTS correspondants :

\begin{tabular}{|l|c|}
\hline \multicolumn{1}{|c|}{ Nom DU MODULE OU DU COURS } & NOMBRE D'ECTS \\
\hline Communication interlinguistique & 5 \\
\hline Communication institutionnelle pour un public étranger & 7 \\
\hline $\begin{array}{l}\text { Techniques et ressources pour la traduction et l'interprétation } \\
\text { dans les services publics }\end{array}$ & 5 \\
\hline $\begin{array}{l}\text { Interprétation dans le domaine de la santé } \\
\text { (propre à chaque combinaison linguistique) }\end{array}$ & 8 \\
\hline $\begin{array}{l}\text { Interprétation dans le domaine juridico-légal et administratif } \\
\text { (propre à chaque combinaison linguistique) }\end{array}$ & 5 \\
\hline $\begin{array}{l}\text { Traduction spécialisée : domaine de la santé } \\
\text { (propre à chaque combinaison linguistique) }\end{array}$ & 5 \\
\hline $\begin{array}{l}\text { Traduction spécialisée : domaine juridique } \\
\text { (propre à chaque combinaison linguistique) }\end{array}$ & 5 \\
\hline $\begin{array}{l}\text { Traduction spécialisée : domaine administratif } \\
\text { (propre à chaque combinaison linguistique) }\end{array}$ & 5 \\
\hline $\begin{array}{l}\text { Stages en entreprises et dans des institutions } \\
\text { (propre à chaque combinaison linguistique) }\end{array}$ & 9 \\
\hline Mémoire de Master & 6 \\
\hline
\end{tabular}

En ce qui concerne la recherche en TISP, le réseau COMUNICA est sans doute la meilleure source d'information qui soit, puisqu'il est constitué de chercheurs qui se consacrent spécifiquement à la TISP. II s'agit des groupes GRETI, CRIT, FITISPos, MIRAS, ALFAQUEQUE et FILSE.

(14) ECTS : Système européen de transfert et d'accumulation de crédits. 
Tous travaillent activement dans ce domaine et collaborent entre eux. Parmi leurs résultats, citons :

- la réalisation de projets de recherche et de développement sur la qualité de la communication avec des populations étrangères permettant de réunir des données de travail objectives;

- l'élaboration de matériaux multilingues sur la communication interculturelle. Parmi les publications les plus récentes : Guía multilingüe de atención al menor, Guía multilingüe de Pediatría, Guía multilingüe de atención a mujeres embarazadas, Guía multilingüe de atención al inmigrante en los servicios sociales(15) (tous ces manuels ont été systématiquement publiés en arabe, bulgare, chinois, français, anglais, polonais, roumain et russe, en plus de l'espagnol, par le groupe FITISPos) ;

- des publications et projets sur la médiation interlinguistique ;

- la rédaction de thèses de doctorat en TISP ;

- la participation à des projets européens (GROTIUS, AGIS, BUILDING MUTUAL, TRUST, EULITA, OPTIMALE, etc.).

Toutefois, je vous invite à consulter les sites web de ces groupes afin de vous informer davantage sur l'ampleur de notre travail dans le domaine de la TISP. Quant à la professionnalisation du T\&l dans les services publics, il est essentiel de rechercher une reconnaissance de son travail par les institutions et par la société en général et, à ce sujet, nous nous permettons d'esquisser brièvement les actions suivantes:

- Recherche d'une collaboration entre des structures d'enseignement supérieur et diverses institutions publiques et privées pour que les étudiants en formation puissent effectuer des stages sur le terrain

- Création d'un concours public pour accéder aux postes d'interprétation téléphonique dans le domaine de la santé

- Collaboration avec des centres spécialisés dans la formation de médiateurs interculturels, tels que l'EPIC(16) ou le SEMSI(17)

- Création et proposition de matériels plurilingues pour lesquels la collaboration de T\&l spécialisés est de plus en plus sollicitée

- Mise en place de programmes pour l'immigration, avec différentes activités qui facilitent le contact entre les populations étrangères et les institutions

(15) Guide multilingue de prise en charge du mineur, Guide multilingue de pédiatrie, Guide multilingue de prise en charge des femmes enceintes, Guide multilingue de prise en charge de l'immigrant dans les services sociaux.

(16) École des Professionnels de l'Immigration et de la Coopération.

(17) Service de Médiation Sociale Interculturelle. 
- Augmentation de l'offre de formation et du nombre de contrats pour les médiateurs interculturels et interlinguistiques dans plusieurs institutions

- Création, dans diverses mairies, d'un réseau de Traducteurs et Interprètes sélectionnés sur concours ou examen.

\section{Configuration de la chaîne de communication : trois maillons dans le processus de communication}

Ce bref rappel de la situation de la communication avec le public non hispanophone en Espagne nous laisse penser que la dernière décennie a connu des progrès, certes significatifs, mais encore insuffisants, du fait de la faiblesse des moyens, de l'inégalité entre les Communautés autonomes ou les régions, de la dispersion, de la faible professionnalisation et de l'absence quasi totale de reconnaissance du travail de l'intermédiaire.

Si l'on devait se situer sur l'échelle des phases mentionnées plus haut, je dirais que nous travaillons sur les échelons 2 et 3 , et que le premier, celui de la reconnaissance des besoins en TISP, est (presque) déjà franchi. Voyons maintenant ce que nous montre l'analyse du processus de communication et des agents qui rendent possible une telle communication.

Au préalable, il convient de rappeler ou d'attirer l'attention sur la complexité du fonctionnement du langage que beaucoup de gens, qui parlent pourtant une deuxième langue et se considèrent bilingues, ignorent ou ont tendance à oublier quand ils interviennent en tant que T\&l bénévoles. Sans eux, la communication serait bien souvent impossible mais, dans certains cas, ils la rendent défectueuse, incomplète ou fausse comme le démontrent certaines de nos recherches. Beaucoup de ces T\&l ad hoc s'attachent à la seule compétence grammaticale (qui laisse parfois à désirer), négligeant les compétences textuelle, pragmatique et sociolinguistique qui doivent accompagner toute communication de qualité.

Pour en revenir aux trois maillons de la chaîne (fournisseurs, usagers, T\&l), Ann Corsellis(18) fait remarquer que chacun d'eux est soumis à un processus de changement ; tous ces processus ont lieu parallèlement et sont facilement observables en Espagne.

\subsection{Processus A. Les services publics (ou institutions publiques)}

La liste ci-après présente les étapes successives franchies au sein d'un service public face à une affluence subite de personnes de langues et de cultures différentes:

1. Au départ, pour l'institution concernée, le problème n'existe pas.

(18) 2002: 71-90. 
2. Le problème est escamoté et les rares tentatives de solution sont peu rationnelles, ce qui conduit à ces commentaires tellement rebattus : " en général, nous n'avons pas de problème car ils ont leurs enfants ou leurs amis pour les aider " ; " on se comprend par signes, dessins... " ; " il y a, au centre, des gens qui peuvent dépanner en cas de besoin ".

3. Le problème est reconnu et accepté.

4. Des solutions ad hoc sont analysées et mises en place.

5. Des progrès sont faits, grâce à des projets réalisés au niveau local, issus d'initiatives personnelles ou privées, malgré de grandes difficultés.

6. Institutionnalisation au niveau national, généralement accompagnée de modifications législatives dictées par la situation.

7. Cohérence, au niveau local, et ceci dans un cadre national, obtenue par la mise en œuvre de mesures générales : règlements, services d'interprètes professionnels, etc.

II s'avère souvent beaucoup plus difficile, et plus long, de mettre en pratique les différentes étapes mentionnées que de les écrire noir sur blanc. Comme le soulignait Corsellis(19), il faut non seulement compter sur des professionnels pour mener à bien les tâches d'interprétation et de traduction, mais aussi former son propre personnel pour qu'il soit capable de travailler avec des gens issus d'autres cultures ou avec des interprètes et des traducteurs.

Dans le cas de l'Espagne, par exemple, on note qu'un plus grand nombre de centres, de nature diverse (mairies, services sociaux, centres éducatifs, hôpitaux...), offrent des services de T\&l en plus de l'accompagnement classique par les parents ou amis. De même, certains ministères ou institutions régionales font appel à des entreprises privées pour la fourniture de prestations de T\&l. Mais il faut ajouter à cela les plaintes des T\&l fonctionnaires eux-mêmes, sur la qualité des services proposés et leurs revendications quant à la réforme des pré-requis exigés pour accéder à ce type de poste. Tout cela prouve que les choses bougent.

\subsection{Processus B. Les intermédiaires}

Intéressons-nous maintenant aux intermédiaires ou aux personnes qui rendent possible la communication lorsqu'il s'agit de langues distinctes, qu'ils soient appelés traducteurs, interprètes, médiateurs, experts linguistiques ou accompagnateurs bénévoles.

Ces personnes sont généralement confrontées à différentes phases, qui sont :

1. Négation de l'existence du problème (ce constat s'applique aussi bien aux intermédiaires qu'aux institutions)

2. Reconnaissance du problème et mise en place de solutions

3. Processus de régularisation de la profession.

(19) 2005: 313-318. 
Aujourd'hui, des voix s'élèvent pour défendre l'inscription de cette activité au registre des professions reconnues par l'État, tandis que la complexité de l'activité et la variété de ses appellations sont reconnues. On dispose d'une meilleure offre de formation.

En ce sens, il convient de souligner l'élaboration d'un Livre Blanc de la Traduction Institutionnelle(20) auquel participent les différents ministères ainsi que d'autres institutions, et dont l'objectif fondamental consiste à proposer un nouveau modèle d'organisation pour la T\&l institutionnelle en Espagne, basé sur les services existants, qu'ils soient communs ou propres à chaque ministère, et dirigés par des professionnels qui connaissent les besoins et les spécificités de la T\&l.

Cette proposition, sous l'égide de la T\&l institutionnelle, englobe principalement les domaines diplomatique, judiciaire, pénitentiaire ainsi que celui des forces de l'ordre, alors que d'autres sphères (santé, éducation), qui appartiennent également à la TISP, en seraient exclues. II s'agit néanmoins d'un pas important.

\subsection{Processus C. Les usagers}

II manque, dans ce processus, les usagers qui ne dominent pas ou qui ignorent totalement la langue de communication. II faut, dans ce cas, s'intéresser à l'individu plus qu'à des grands groupes, pour éviter les stéréotypes et pour tenir compte du fait que chaque cas est différent. Par conséquent, plutôt que de définir un processus (figé) pour un groupe, on peut esquisser une démarche adaptable aux variables en jeu et qui tient compte de facteurs antérieurs à la migration (éducation, conditions de vie, expérience professionnelle, liens familiaux), contemporains de la migration (planification du voyage, expérience vécue, type de migration) et postérieurs à la migration (degré de traumatisme, capacité à surmonter les barrières culturelles, connaissance de la langue du pays d'accueil ou degré d'adaptation à la nouvelle société) sans oublier la dimension des générations futures.

Ces générations futures seraient-elles la solution ? L'histoire semble prouver le contraire puisque des pays qui, depuis plusieurs générations, accueillent des personnes parlant des langues différentes continuent à avoir besoin de T\&l et de médiateurs, tandis que beaucoup de jeunes ont cessé d'utiliser la langue de leurs parents et ignorent presque tout de la culture de leurs ancêtres.

Corsellis conclut(21) en disant que les conditions de réussite sont uniquement réunies quand il existe une relation horizontale optimale entre ces trois processus verticaux. Nous sommes, pour l'instant, sur la bonne voie mais le chemin à parcourir est encore long et un travail d'équipe s'impose.

(20) http://ec.europa.eu/spain/pdf/libro blanco traduccion es.pdf

(21) $2002: 71-90$

Traduire $\mathrm{n}^{\circ} 227$, décembre 2012 


\section{Conclusions}

En résumé et à la lumière de ma propre expérience en tant que chercheuse, formatrice et professionnelle de la T\&l, je dirais qu'il reste des questions encore en suspens avant de pouvoir passer à l'étape définitive. En voici quelques-unes :

1. Nécessité de définir le profil du T\&l dans les services publics, de déterminer ses compétences et de faire reconnaître son travail

2. Nécessité de créer une infrastructure appropriée, ce qui suppose donc l'attribution d'un budget pour ne pas dépendre, comme c'est le plus souvent le cas, de subventions annuelles ou ponctuelles

3. Nécessité de faire prendre conscience à la société et aux institutions qu'il faut encore développer des mécanismes pour permettre cette communication par des professionnels dont le niveau est équivalent à celui de l'interprète de conférence ou du traducteur technique. Cela entraînerait la création de postes, avec une rémunération adaptée.

Pour y arriver, il faut continuer à travailler et à mettre en place des stratégies précises, par exemple :

1. Multiplier les séminaires, cours, ateliers ou journées destinés aux personnels des services publics. Leur fonction doit les amener à réfléchir à la qualité de la communication. II faut également intégrer à leur préparation la participation aux jeux de rôle et aux cours organisés pour les futurs interprètes ou leur fournir un enseignement spécifique sur la manière de travailler avec des interprètes ; ce sont là quelques-unes des méthodes qui ont donné les meilleurs résultats

2. Développer plus de programmes adaptés en TISP où l'on prendrait en compte les besoins et compétences que doit acquérir ou maîtriser le professionnel de la communication interlinguistique

3. Consolider le travail en équipe. Franz Pöchhacker (2008), lors de la conférence de clôture du Troisième Congrès International sur la TISP organisé à l'Université d'Alcalá de Henares en avril 2008, a insisté sur la nécessité d'une interaction entre la formation, la recherche et les relations avec les institutions, le tout reposant sur trois piliers : autorité, crédibilité et confiance mutuelle. Cela permettrait la mise en place de solutions interdisciplinaires telles que les recherches conjointes, la réalisation de mesures communes ou le développement de politiques consensuelles sur des thèmes clés tels que la définition du profil professionnel du T\&l

4. Mettre en œuvre les décisions adoptées par l'Union européenne, qui a récemment pris conscience que les droits des citoyens pouvaient être lésés dans les États membres si l'on ne cherchait pas une formule égalitaire pour tous. D'où le Forum de réflexion sur le plurilinguisme et la formation des interprètes organisé par la Commission Européenne et la pro- 
position d'une directive relative au droit à l'interprétation et à la traduction, nouvellement adoptée, ou encore le lancement du groupe SIGTIPS(22).

Certaines de ces mesures sont déjà en place, mais elles restent insuffisantes. Quant à la formation, on continue à hésiter, dans certains milieux en Espagne, entre deux types de traducteur/interprète, l'un qui interviendrait de façon neutre, l'autre qui assurerait une plaidoirie, plus proche de la médiation. Dans le premier cas, l'interprète est invisible, alors que dans le second il est là, littéralement, pour se faire l'avocat de l'usager (ou de l'une des parties) et prendre position, formuler ses propres questions, conseiller, et même livrer son opinion personnelle sur les sujets dont il est question.

En ce qui concerne la recherche en TISP, les progrès obtenus doivent désormais influer sur la pratique sociale, en passant de la recherche théorique à la recherche appliquée en milieu réel : infirmerie, services sociaux, écoles, commissariats, de manière à favoriser le développement d'un modèle de qualité applicable à ces pratiques. N'oublions pas d'ailleurs qu'une bonne pratique exige de bons professionnels.

On pourrait résumer ainsi la situation actuelle de l'Espagne par rapport à la TISP :

- succès que représente l'intérêt grandissant porté à la TISP dans le champ des études de traduction et d'interprétation et des études de communication,

- impact limité dans d'autres disciplines : la médecine, le droit, le travail social...

- rapports déséquilibrés entre recherche et pratique,

- développement inachevé d'une politique cohérente homogène.

Pour ce dernier point et en guise de conclusion, il convient de souligner que la politique migratoire de chaque pays a une très forte incidence dans ce domaine, bien qu'on ne puisse pas encore parler de modèle unique.

Dans un article publié dans El País(23) intitulé "¿Multiculturaliqué? " (Sánchez Vallejo)(24) et portant comme sous-titre "L'intégration des immigrants dans l'Union européenne provoque de fortes tensions ", Angela Merkel reconnaît l'échec du modèle de diversité culturelle en Allemagne. Et Carlos Giménez, anthropologue et professeur à l'Université Autonome de Madrid, commente ainsi le système espagnol (multicuraliste ou interculturaliste, qui favorise une cohabitation plus active) : " Nous avons un modèle très original. II s'agit du Plan de citoyenneté et d'intégration, selon lequel nous sommes tous des citoyens, avec des droits et des devoirs ". Un modèle qui, selon l'anthropologue, se résume à " une citoyenneté commune et la cohabitation des cultures".

(22) Special Interest Group on Translation and Interpreting for Public Services.

(23) Dimanche 24 octobre 2010 : 32-33.

(24) "Multiculturel talors?". 
Toutefois, ce débat s'écarte du thème de cette communication, même s'il peut nous pousser à réfléchir aux mécanismes activés pour mener à bien une politique d'intégration ou d'assimilation et aux répercussions (éventuelles) sur la reconnaissance et la professionnalisation de la TISP.

Dans le monde globalisé d'aujourd'hui, où tout se partage (y compris la crise), il me semble que seuls la collaboration et l'échange de matériels et d'initiatives liés à la TISP permettront désormais de réaliser de vrais progrès.

carmen.valero@uah.es

Article traduit par Géraldine Galeote et les étudiantes d'espagnol du Master 1 T3L de I'Université Paris 8 (Rosalía Barcia, Anne-Charlotte Chasset, Béatrice Cornière, Aurélie Dubois, Pauline Louvet, Viviane Petit).

\section{Bibliographie}

Brunette, L., G. Bastin, I. Hemlin, et H. Clarke, eds. 2003. The Critical Link 3: Interpreters in the Community. Selected Papers from the Third International Conference on Interpreting in Legal, Health and Social Service Settings. Amsterdam, Pays-Bas/Philadelphia, États-Unis : Benjamins.

CarR, S., RoberTs, R., Dufour, A. et Steyn, D. 1997. The Critical Link: Interpreters in the Community. Amsterdam, Pays-Bas : Benjamins.

CORSELLIS, A. 2002. " Formación de los proveedores de servicios públicos para trabajar con intérpretes y traductores. Habilidades y competencias interculturales ". Traducción e Interpretación en los Servicios Públicos. Contextualización, actualidad y futuro. Ed. Carmen ValeroGarcés. Granada, Espagne : Comares : 71-90.

CORSELLIS, A. 2005. "Who Takes Responsibility for What in the Intercultural, Interlingual Exchange? ", et Traducción como mediación entre lenguas y culturas / Translation as Mediation or How to Bridge Linguistic and Cultural Gaps. Ed. C. Valero-Garcés. Alcalá de Henares Servicio de Publicaciones de la Universidad : 313-318.

MIKKELSON, H. 2001. Interpreting is Interpreting - Or is it? [Document Internet disponible sur : http://www.acebo.com/papers/interp1.htm].

PöCHHACKER, F. 2002. "Getting organized: The evolution of community interpreting", Interpreting, 4, 1, pp. 125-140.

PöCHHACKER, F. 2008. "Alcalá III: On Balance, And Off", Conférence de clôture prononcée à l'Université d'Alcalá et lors du 3e Congrès International de la TISP, 25 avril 2008. 
Roberts, R., Carr, S. Abraham, D. et Dufour, A. Eds. 2000. The Critical Link 2: Interpreters in the Community. Amsterdam, Pays-Bas/Philadelphia, États-Unis : Benjamins.

SÁnchez-Vallejo, A. 2010. "¿Multiculturaliqué? ", El País (dimanche 24 octobre 2009, pp. 32 33.

VALERO GARCÉS, C. 1998. "¿Traducción e interpretación en servicios públicos? ¿De qué me hablas? ¿Una nueva especialización? ". En Nuevas Tendencias y Aplicaciones de la Traducción., C. Valero Garcés, ed. Alcalá de Henares : Servicio de Publicaciones de la Universidad : 267- 277.

VALERO GARCÉS, C. 2003. "Una visión general de la evolución de la Traducción e Interpretación en los Servicios Públicos ". Traducción e interpretación en los Servicios Públicos. Contextualización, actualidad y futuro. Granada, Espagne : Comares, 3-33.

VALERO GARCÉs, C. ed. 2005. Traducción como mediación entre lenguas y culturas/Translation as mediation or how to bridge linguistic and cultural gaps. Alcalá de Henares : Université d'Alcalá.

VALERO GARCÉS, C. ed. 2008. Investigación y práctica en Traducción e Interpretación en los Servicios Públicos: desafíos y alianzas / Research and Practice in Public Service Interpreting and Translation: Challenges and Alliances. Alcalá de Henares : Servicio de Publicaciones de la Universidad.

Valero Garcés, C. et G. Mancho eds. 2002. Traducción e Interpretación en los Servicios Públicos: Nuevas necesidades para nuevas realidades / New Needs for New Realities. Alcalá de Henares : Servicio de Publicaciones de la Universidad.

Wädensjo, C., Englund Dimitrova, B et Nilsson, A. 2007. The Critical Link 4. Amsterdam, PaysBas/Philadelphia, États-Unis : Benjamins.

Carmen Valero Garcés est professeure de traduction et d'interprétation à l'Université d'Alcalá en Espagne. Docteure en philologie anglaise et titulaire d'un master en migration et relations intercommunautaires, elle dirige le programme de formation en traduction et interprétation dans les services publics. Elle organise depuis 1996 les Rencontres internationales de la traduction, dont les 4 dernières éditions $(2002,2005,2008,2011)$ étaient consacrées à la traduction et à l'interprétation dans les services publics.

Entre 1994 et 2002, elle a enseigné la traduction et l'interprétation à l'Université du Minnesota et a collaboré sur plusieurs projets avec les responsables de cette filière. 